\title{
Systematic review and meta-analysis of treating meibomian gland dysfunction with azithromycin
}

\author{
Tianchang $\mathrm{Tao}^{1} \cdot$ Liming $\mathrm{Tao}^{2}$
}

Received: 28 October 2018 / Revised: 27 January 2020 / Accepted: 4 March 2020 / Published online: 28 April 2020

(c) The Author(s), under exclusive licence to The Royal College of Ophthalmologists 2020

\begin{abstract}
To systematically review studies of managing meibomian gland dysfunction (MGD) with azithromycin and pool clinical outcomes to show its effectiveness. Eligible studies were retrieved from five main electronic databases. Symptom score was the primary outcome, while clinical signs and objective measurements were secondary outcomes. Pooled rates for adverse events were also calculated. Improvements in each outcome after administering either oral azithromycin (OA) or topical azithromycin (TA) were pooled and measured by standard mean difference (SMD) to show the overall effectiveness. Then the effectiveness was sub-grouped by TA and OA. In addition, pooled outcomes after administering TA and oral doxycycline (OD) were compared with assess their effectiveness. Finally, 18 eligible studies were included. The overall pooled symptom scores were significantly reduced after administering both TA and OA $[P<0.0001 ; \mathrm{SMD}=1.54$ (95\% CI: 1.15-1.92)]. Similarly, the overall combined eyelid signs, plugging of the meibomian gland, meibum quality, and tear secretion were also distinctly improved. However, significant improvements for tear break-up time (TBUT) and corneal staining $(\mathrm{CS})$ were achieved by TA (TBUT: $P=0.02$; $\mathrm{CS}$ : $\mathrm{P}=0.02$ ) but not by (TBUT: $P=0.08$; CS: $P=0.14$ ). The pooled adverse event rates for TA and OA were $25 \%$ and $7 \%$, respectively. Moreover, TA was comparable to OD to treat MGD regarding symptom score, TBUT and tear secretion. This study showed that MGD could be treated effectively with oral or topical azithromycin by improving symptoms, clinical signs, and stabilization of tear film. Topical azithromycin seemed to be superior over oral azithromycin or doxycycline in improving the quality of tear film in the short term.
\end{abstract}

\section{Introduction}

Meibomian gland dysfunction (MGD) is a very common ocular surface disease with a prevalence of $39-50 \%$ and almost encountered every day by ophthalmologists in their outpatient departments [1]. The disease is a chronic and diffuse abnormality of the meibomian gland that is always accompanied by subsequent blepharitis, chalazion, and meibomitis and is the most common cause for evaporative dry eye syndrome [2]. People who suffer from acne rosacea

Liming Tao

1mtao9@163.com

1 Department of Ophthalmology, Peking University People's Hospital, Eye Diseases and Optometry Institute, Beijing Key Laboratory of Diagnosis and Therapy of Retinal and Choroid Diseases College of Optometry, Peking University Health Science Center, Beijing, China

2 Department of Ophthalmology, The Second Hospital Affiliated to Anhui Medical University, Hefei, Anhui, China or seborrheic dermatitis or psoriasis are more prone to have MGD than the general population [3]. Complicated pathogenesis of the disease combined with the cutaneous conditions makes it hard to manage, while the current means of therapy are limited [4].

To achieve better therapeutic effects, combined means are always employed to treat the disease because both inflammatory and bacterial elements are involved in the development of MGD [5]. To date, eyelid hygiene, lid massage, warm compresses, artificial tears, and topical antibiotics are routine ways to treat the disease [4]. In addition, topical steroids and/or immunosuppressive agents (e.g., cyclosporine A, Cs A) have been used in some cases and gained satisfactory outcomes, but the complications from steroids and high cost of Cs A cannot be ignored for long-term use [6,7]. Considering the pathogenesis of MGD, medications that have dual effects targeting the two elements should be the optimal choice for treatment.

Macrolide antibiotics, which have dual effects, have been indicated to be effective in treating MGD and have been 
the traditional systemic management for acne rosacea and posterior blepharitis, but the severe side effects of firstgeneration macrolides limit their clinical use [8]. Compared with former macrolides, azithromycin is a semi-synthetic macrolide antibiotic of the second generation, which is characterized by a long half-life, good intraocular penetration, and broad antibacterial scope [9-11]. Previous studies have verified prolonged high-level ocular concentrations could be achieved after administering either oral azithromycin (OA) or topical azithromycin (TA); even after discontinuing the medication, relatively high concentrations still can be maintained in the conjunctiva [8, 9]. Based on these advantages, azithromycin, either topical, or oral formulations, has been employed to treat MGD in recent years. Although the efficacy of treating MGD with azithromycin has been testified in several clinical studies, contrary results were found when comparing some outcomes (e.g., tear secretion, corneal staining, and Schirmer test) among these studies [1, 4, 8, 12-26]. In addition, comparisons of managing MGD with different azithromycin formulations and the effectiveness between azithromycin and other macrolides are still not confirmative due to the small sample sizes of the studies. Thus, pooling the data of the current studies by means of meta-analysis seems to be an ideal way to draw relatively reliable conclusions.

The aim of this study is to systematically review the clinical trials on treating MGD with either TA or OA and combine the outcomes by pooling data regarding symptom scores, clinical signs, and objective measurements. In addition, the effectiveness of managing MGD between TA and oral doxycycline (OD) will be compared.

\section{Materials and methods}

\section{Strategy for literature retrieval}

Two independent reviewers searched five electronic databases, PUBMED, EMBASE, OVID Medline, the Cochrane Library, and ClinicalTrials.gov, to retrieve literature on treating MGD with azithromycin. Synonyms for MGD, such as posterior blepharitis, chronic blepharitis, meibomianitis, meibomitis, and ocular rosacea, were also used to expand the search scope, although these terms seem not to be interchangeable [4]. Moreover, alternative terms or acronyms of azithromycin (e.g., macrolide antibiotic, AMZ, and AZI) were employed to avoid missing any related citations. After database searching, the two reviewers also indexed the references of the retrieved literature manually in order to find other related literature or conference proceedings. Finally, the reviewers compared their search results and solved discrepancies with discussion.

\section{Study selection}

The two independent reviewers selected target literature mainly based on participants (patients with MGD) and interventions (oral and/or topical azithromycin). Briefly, they excluded obvious unrelated literature by scanning titles. Then, eligible literature was selected through browsing abstracts and full-text versions of articles. The reviewers compared and discussed the selected results until a consensus was achieved.

\section{Studies, participants, and interventions}

All studies about treating MGD with azithromycin were included without considering what the study design or azithromycin formulation was. Patients with MGD combined with posterior blepharitis, ocular rosacea, or seborrheic dermatitis were the participants. According to the definition of MGD proposed by the international workshop, the disease was defined as a diffuse and chronic abnormality of the meibomian gland and characterized by terminal duct obstruction and/or qualitative or quantitative changes in meibum [2]. Patients $<18$ years or women of child-bearing age or who were breastfeeding were excluded due to the possible side effects of azithromycin.

\section{Clinical outcomes}

Improvement in symptom score at the end of follow-up was chosen as the primary outcome in the present study because most patients with MGD complain of symptoms linked to the ocular surface which affect their daily life severely, including foreign body sensation, ocular itching, eye redness, dry eye intolerance, etc. In addition, clinical signs that occurred frequently and objective measurements were set as the secondary outcomes. Clinical signs included eyelid signs (lid debris, lid swelling, and lid redness), plugging of the meibomian gland, meibum quality, and conjunctival injection. Objective measurements encompassed in this study were corneal staining, tear break-up time (TBUT), lissamine green staining, and tear secretion (Schirmer test I/II). In addition, adverse events were also recorded.

\section{Data extraction and management}

Study characteristics of each eligible study, including study design, region, sample size, demographics of the participants (age and gender ratio), interventions (formulation, dose, frequency, and duration), supplemental treatments, follow-up, and the clinical outcomes were all extracted and managed by the two reviewers independently. The extracted data were compared between the reviewers, and discrepancies were solved by discussion or by consulting a third reviewer. 


\section{Study quality assessment}

The included studies were assessed by a 14-item checklist (http://links.lww.com/ICO/A265) developed by the Review Body for Interventional Procedures. The checklist could be used for evaluating either prospective or retrospective studies, and these 14 items cover the main elements of an interventional study. Each item is an assessment question that could be answered by three options: "Yes" (Y), "No" $(\mathrm{N})$, and "Unclear" (U). One study could be deemed as high-quality when at least 8-9 "Yes" responses were obtained. According to the checklist, important prognostic factors (Item 5) defined in this study included participant gender and age, disease severity, combined conditions (e.g., posterior blepharitis, rosacea, and seborrheic dermatitis), disease course and previous treatments (e.g., lid hygiene, lid message or topical administrations), and important outcomes (Item 11) were symptom scores, eyelid signs and/or one of the objective measurements. Moreover, a long follow-up period (Item 12) was defined as $>1$ month.

\section{Effect size and statistical analyses}

In this study, all the statistical analyses, including randomeffects model and fixed-effects model, were performed by using Stata 14.0 (Stata Corp LLC, College Station, TX, USA). The outcomes were assessed by standard mean difference (SMD) rather than mean difference (MD) for reducing the heterogeneity generated from different grading systems adopted in each study. Improvements in each outcome after administering either $\mathrm{OA}$ or TA were pooled together to evaluate the overall effectiveness. Then, the effectiveness for some important outcomes (e.g., symptom score, TBUT, and corneal staining) was sub-grouped by $\mathrm{TA}$ and $\mathrm{OA}$ to demonstrate the influences of the different formulations on the effectiveness. To compare the effectiveness between TA and OD, the outcomes extracted from the comparative studies were combined. In addition, occurrence rates of adverse events for either TA or OA were calculated, respectively. All the effect size values were expressed as a mean and $95 \%$ confidence interval $(\mathrm{CI})$. When distinct heterogeneity $(P$ value for $\mathrm{Chi}^{2}$ test $<0.1$ or $\mathrm{I}^{2}>50 \%$ ) existed, the random-effects model was used, but when distinct heterogeneity was not present, the fixed-effects model was employed. $P<0.05$ was considered as the significant threshold.

\section{Results}

\section{Study selection}

Figure 1 shows the diagram of screening eligible studies. A total of 169 records were harvested after searching the

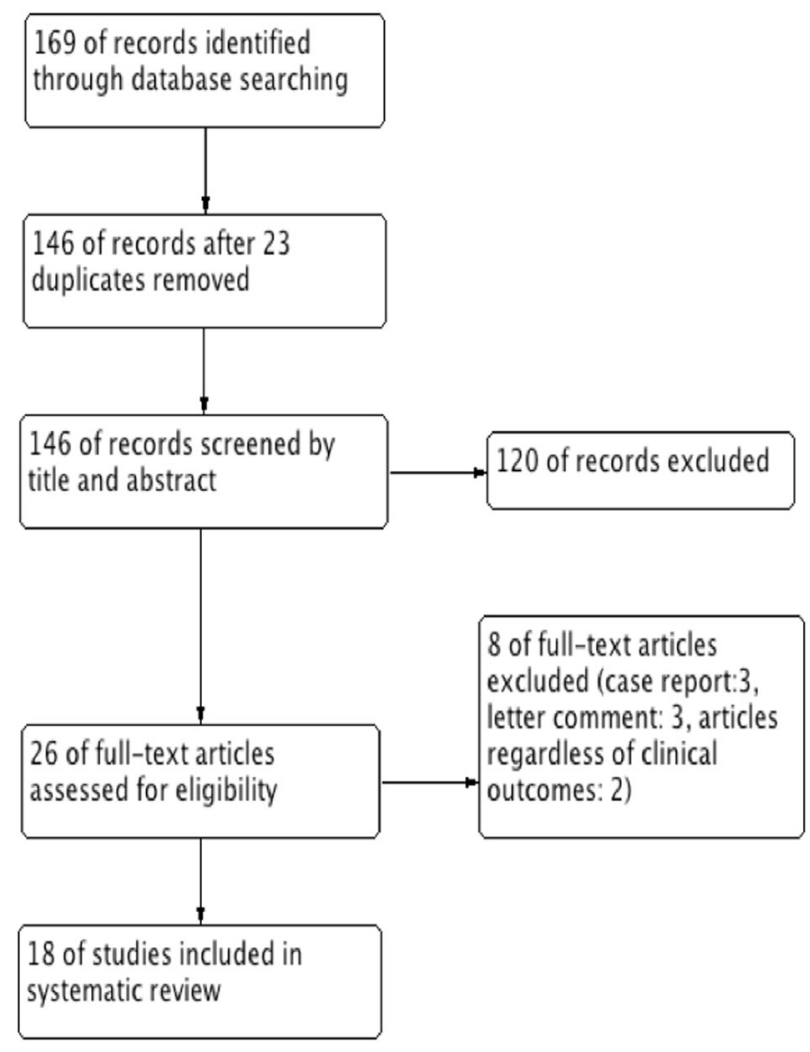

Fig. 1 Flow diagram showing study inclusion and exclusion. Eighteen eligible studies were selected for systematic review and meta-analysis.

databases and indexing the references of the searched literature. After 23 duplicates were removed, 146 records were screened by browsing titles and abstracts. Afterward, 26 records remained. Then, the remaining records were evaluated by their full-text versions, and 18 final studies were included for systematic review and meta-analysis.

\section{Characteristics of included studies}

Table 1 presents the characteristics of the included studies. The publication year of these studies spanned from 2008 to 2018. The regions covered nine countries with different races. Among the 18 studies, seven studies (38.9\%) were random clinical trials (RCTs), while the others $(61.1 \%)$ were non-randomized studies (seven were prospective studies and four were retrospective studies). Referring to the presence of a control group, nine (50.0\%) were non-comparative studies; the others $(50.0 \%)$ were comparative studies, including topical azithromycin versus oral doxycycline/azithromycin $(n=5,27.8 \%)$, oral azithromycin versus oral doxycycline $(n=2,11.1 \%)$, and topical azithromycin versus warm compress or placebo $(n$ $=2,11.1 \%)$. All the patients in the 18 studies suffered from MGD with or without other combined conditions (posterior blepharitis and acne rosacea), but the severity of 


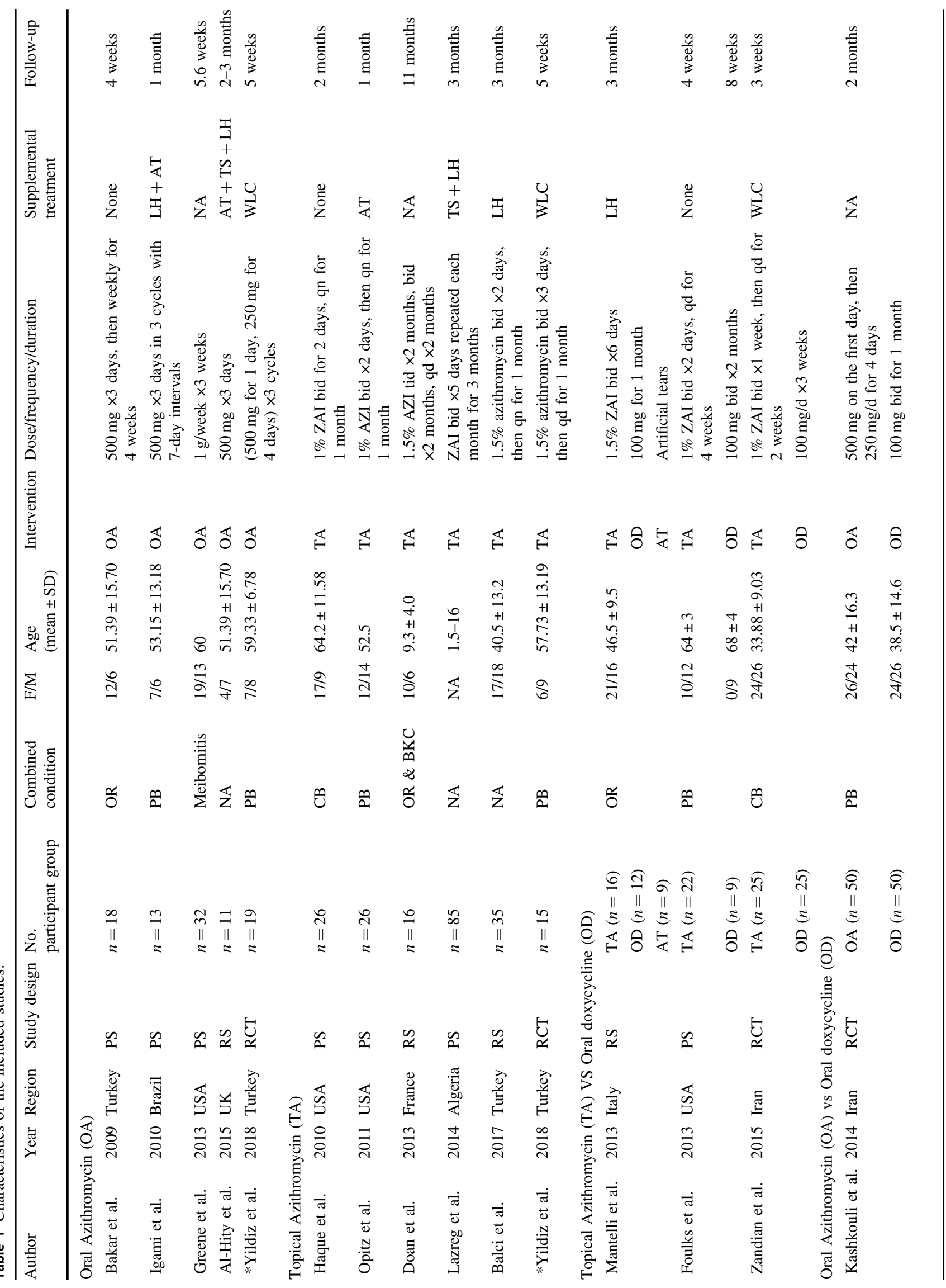




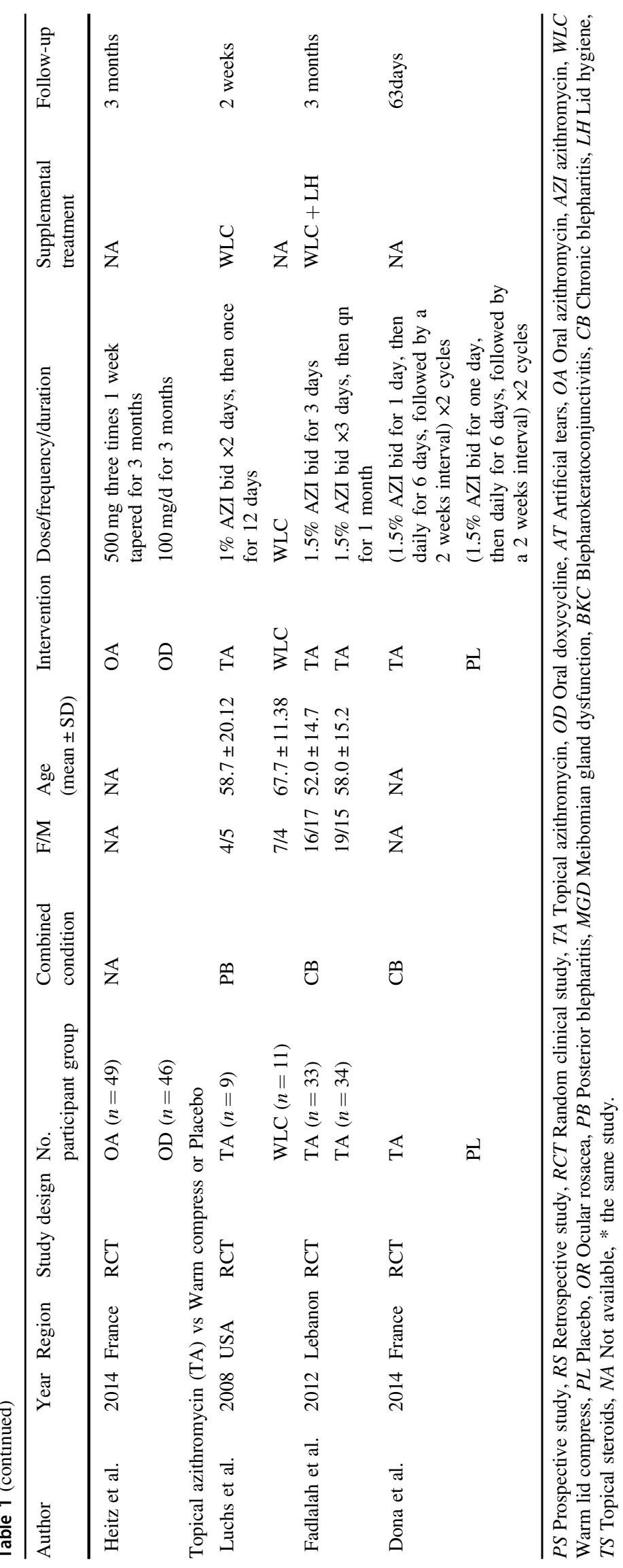




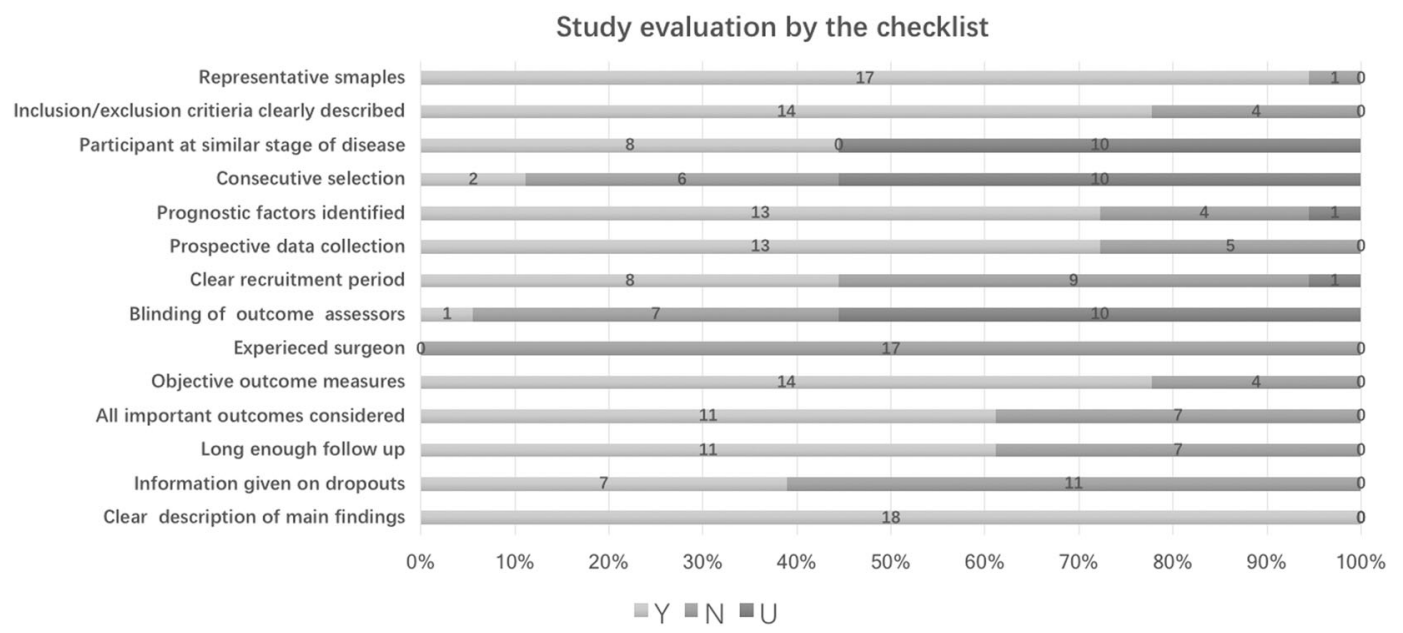

Fig. 2 Evaluation of studies based on the checklist. Most studies were deemed as hight-quality.

MGD varied within these studies. Moreover, azithromycin regimens in the studies varied to some extent. Specifically, TA concentrations applied in these studies were $1.0 \%$ or $1.5 \%$, while single doses of oral azithromycin varied from 250 to $1000 \mathrm{mg}$. Accordingly, the administration frequencies and durations also varied among these studies, and the follow-up durations ranged from 2 weeks to 3 months.

\section{Study assessment}

Figure 2 shows the results of assessing the studies based on the checklist. Among the 18 studies, thirteen (72.2\%) obtained more than 8 "Yes" responses and were deemed as high-quality.

\section{Effectiveness of treating MGD with azithromycin}

Figure 3 shows the overall pooled symptom scores at the end of follow-up were significantly reduced compared with the baseline scores $(Z=7.89, P<0.0001 ; \mathrm{SMD}=1.54$, 95\% CI: 1.15-1.92). As Fig. 4 shows, when the scores were sub-grouped by the different formulations, the pooled scores also showed significant reductions from the baseline scores either after administering $\mathrm{OA}(\mathrm{Z}=4.15, P<0.0001$; $\mathrm{SMD}=1.64,95 \% \mathrm{CI}: 0.87-2.42)$ or TA $(\mathrm{Z}=6.24, P<$ $0.0001, \mathrm{SMD}=1.49,95 \%$ CI: $1.02-1.96$ ).

Table 2 shows the pooled secondary outcomes after administering azithromycin. Regarding the eyelid signs, the pooled lid debris $(\mathrm{Z}=3.56, P<0.0001 ; \mathrm{SMD}=1.53,95 \%$ CI: 0.69-2.37), lid swelling $(Z=2.84, P<0.0001 ; \mathrm{SMD}=$ 1.67, 95\% CI: $0.52-2.82)$ and lid redness $(Z=3.06, P=$ $0.002 ;$ SMD $=1.52,95 \%$ CI: $0.55-2.50)$ improved distinctly by applying azithromycin. As Fig. 5 shows, plugging of the meibomian gland also significantly improved after using either TA $(Z=3.69, P<0.0001 ; \mathrm{SMD}=1.64,95 \%$
CI: $0.77-2.51)$ or $\mathrm{OA}(\mathrm{Z}=3.40, P=0.001$; $\mathrm{SMD}=1.88$, 95\% CI: 0.80-2.96). In addition, both meibum quality $(Z=$ 2.91, $P=0.004 ; \mathrm{SMD}=2.15,95 \%$ CI: $0.70-3.60)$ and conjunctival injection $(Z=3.88, P<0.0001$; $\mathrm{SMD}=0.97$, 95\% CI: $0.48-1.46)$ were also improved substantially by administering azithromycin.

As for objective measurements, the combined scores of lissamine green staining for both formulations were also significantly reduced by administering azithromycin $(Z=$ $1.96, P=0.05 ; \mathrm{SMD}=0.92,95 \% \mathrm{CI}: 0.00-1.85)$. The combined corneal staining after applying TA significantly improved compared with the baseline values $(Z=2.36$, $P=0.02 ; \mathrm{SMD}=0.69,95 \%$ CI: $0.12-1.27)$, but no significant improvements after administering OA were found $(Z=1.47, P=0.14 ;$ SMD $=0.57,95 \%$ CI: $-0.19-1.33)$ (Fig. 6). As another measurement for evaluating the quality of tear film, the pooled TBUT also increased distinctly from baseline values after applying TA $(Z=2.41, P=0.02$; $\mathrm{SMD}=-1.20,95 \% \mathrm{CI}:-2.17$ to -0.22$)$ but not after administering $\mathrm{OA}(Z=1.74, P=0.08 ; \mathrm{SMD}=-0.35,95 \%$ CI: -0.75-0.04) (Fig. 7). However, tear secretion distinctly increased after applying both TA $(Z=2.56, P=0.01$; $\mathrm{SMD}=-0.34,95 \% \mathrm{CI}:-0.60$ to -0.08$)$ and $\mathrm{OA}(Z=$ $2.52, P=0.01 ; \mathrm{SMD}=-0.44,95 \% \mathrm{CI}:-0.79$ to -0.10 ) (Fig. 8).

\section{Comparison between topical azithromycin and oral doxycycline}

The combined improvements in symptom scores between TA and OD showed a significant difference $(Z=0.24, P=$ $0.81 ;$ SMD $=-0.08,95 \%$ CI: $-0.76-0.59)$. Similarly, no distinct differences were found after administering the two medications regarding TBUT $(Z=0.42, P=0.68$; SMD $=$ $0.11,95 \% \mathrm{CI}:-0.43-0.65)$ and tear secretion $(Z=0.00$, $P=1.00 ; \mathrm{SMD}=0.00,95 \%$ CI: $-0.45-0.45)$. 
Study

ID udy

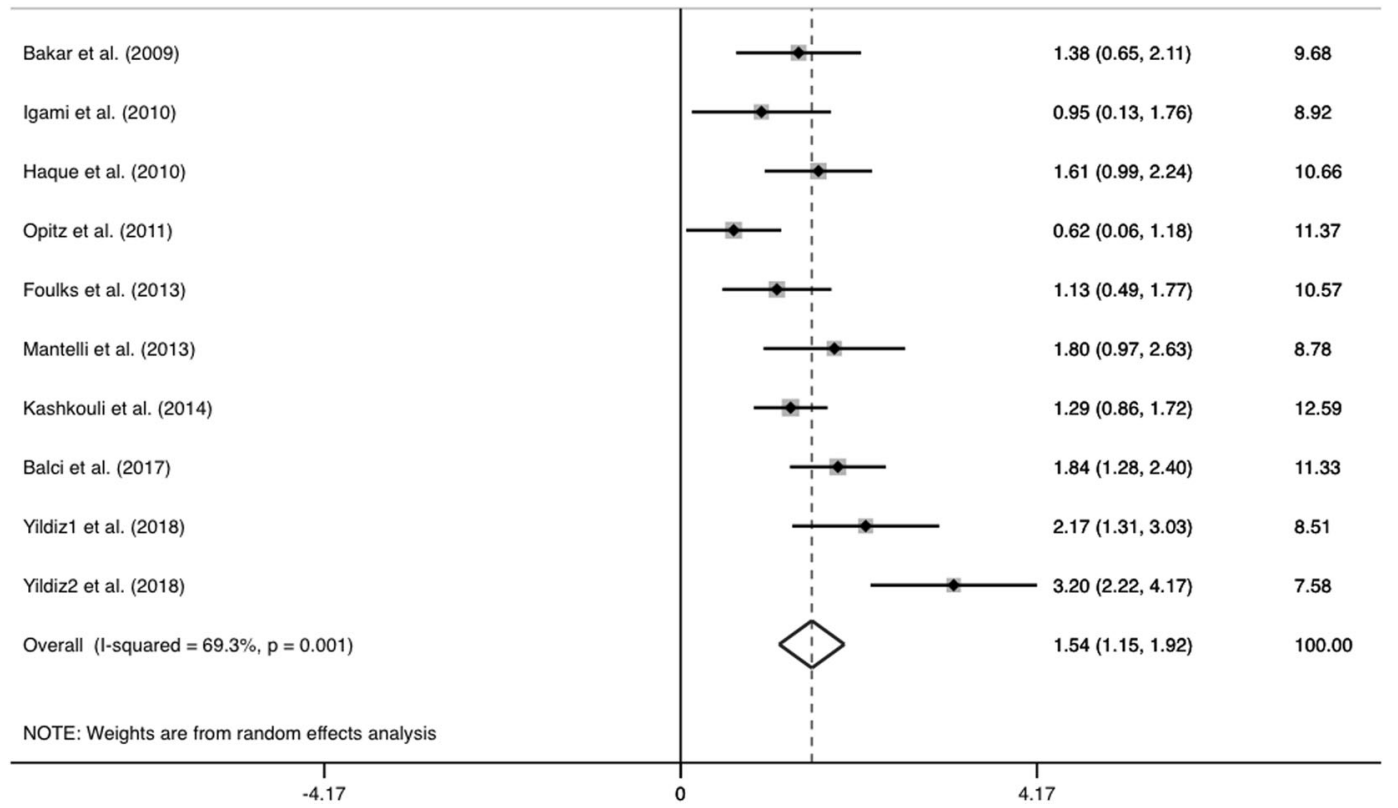

Weight

Fig. 3 Forest plot showing the effect of Azithromycin on symptom scores in MGD patients, ordered by date of publication. The overall pooled symptom scores at the end of follow-up were significantly reduced compared with the baseline.

$$
\text { Study }
$$

ID
$\%$

$\operatorname{SMD}(95 \% \mathrm{Cl}) \quad$ Weight

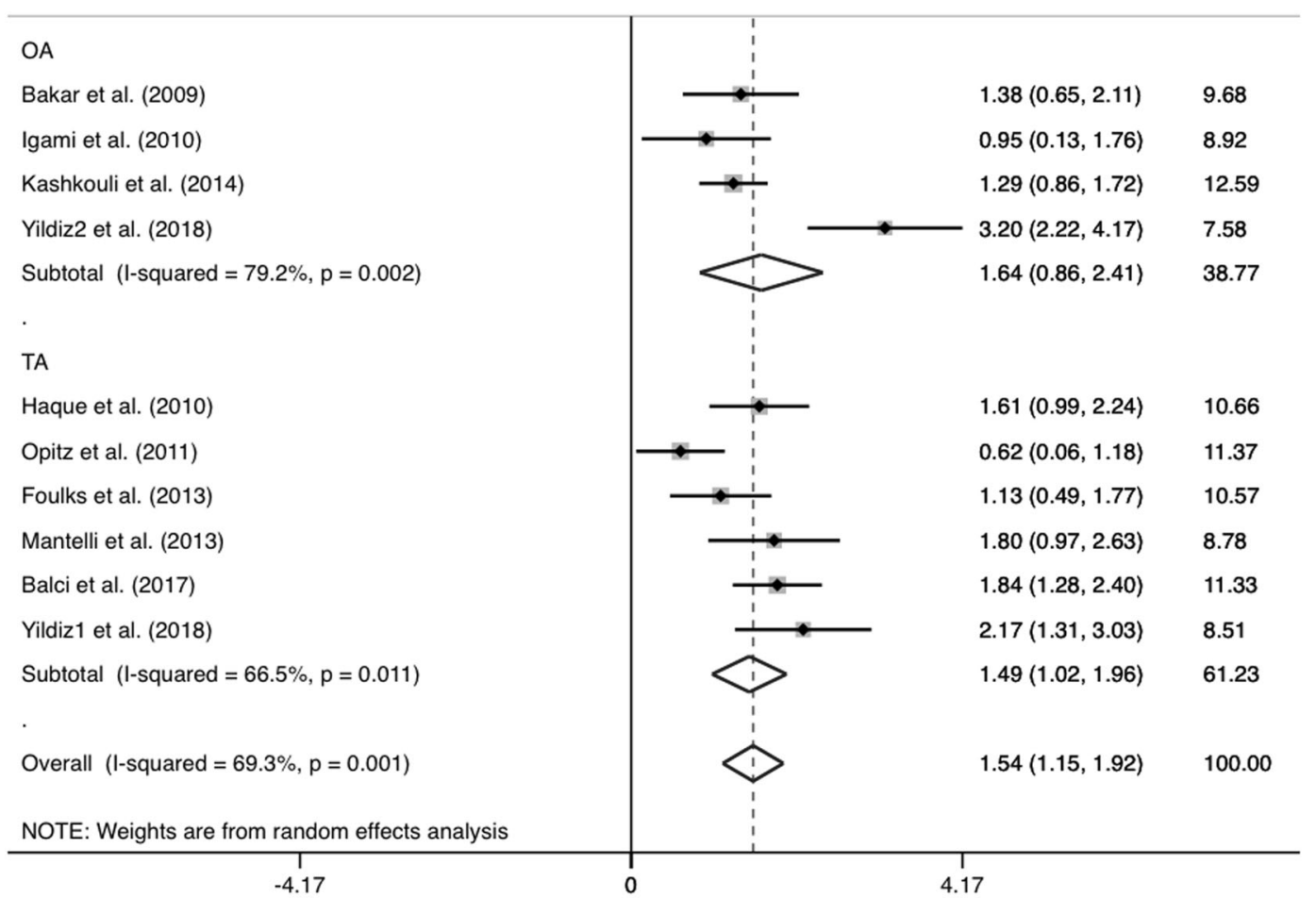

Fig. 4 Forest plot of subgroups showing the effect of Azithromycin on symptom scores in MGD patients (OA or TA), ordered by date of publication. The pooled scores significantly reduced from the baseline after administering either OA or TA. 
Table 2 Pooled secondary outcomes after administering azithromycin.

\begin{tabular}{lcllll}
\hline Outcomes & SMD $(95 \% \mathrm{CI})$ & Heterogeneity $\left(\mathrm{I}^{2}\right)$ & $\mathrm{Z}$ & $P$ & Effects model \\
\hline Lid Deris & $1.53(0.69-2.37)$ & $82.0 \%$ & 3.56 & $<0.0001^{*}$ & Random \\
Lid swelling & $1.67(0.52-2.82)$ & $88.7 \%$ & 2.84 & $0.005^{*}$ & Random \\
Lid redness & $1.52(0.55-2.50)$ & $76.6 \%$ & 3.06 & $0.002^{*}$ & Random \\
Plugging of MG & $1.71(1.05-2.37)$ & $87.8 \%$ & 5.10 & $<0.0001^{*}$ & Random \\
& $1.64(0.77-2.51)$ & $89.0 \%$ & 3.69 & $<0.0001^{* *}$ & Random \\
Meibum quality & $1.88(0.80-2.96)$ & $84.7 \%$ & 3.40 & $0.001^{* * *}$ & Random \\
Conjunctival injection & $2.15(0.70-3.60)$ & $89.4 \%$ & 2.91 & $0.004^{*}$ & Random \\
Corneal staining & $0.97(0.48-1.46)$ & $61.9 \%$ & 3.88 & $<0.0001^{*}$ & Random \\
& $0.64(0.20-1.08)$ & $77.1 \%$ & 2.87 & $0.004^{*}$ & Random \\
Tear break-up time & $0.69(0.12-1.27)$ & $74.3 \%$ & 2.36 & $0.02^{* *}$ & Random \\
& $0.57(-0.19-1.33)$ & $83.4 \%$ & 1.47 & $0.14^{* * *}$ & Random \\
& $-0.90(-1.56$ to -0.25$)$ & $88.5 \%$ & 2.71 & $0.007^{*}$ & Random \\
Lissamine green & $-1.20(-2.17$ to -0.22$)$ & $92.4 \%$ & 2.41 & $0.02^{* *}$ & Random \\
Tear secretion & $-0.35(-0.75-0.04)$ & $0 \%$ & 1.74 & $0.08^{* * *}$ & Random \\
& $-0.92(0.00-1.85)$ & $83.1 \%$ & 1.96 & $0.05^{*}$ & Random \\
& $-0.34(-0.58$ to -0.17$)$ & $6.3 \%$ & 3.56 & $<0.0001^{*}$ & Fixed \\
\hline On -0.08$)$ & $10.1 \%$ & 2.56 & $0.01^{* *}$ & Fixed \\
& $-0.44(-0.79$ to -0.10$)$ & $22.5 \%$ & 2.52 & $0.01^{* * *}$ & Fixed \\
\hline
\end{tabular}

*Overall $P$ value; **sub-grouped $P$ value of topical azithromycin; ***sub-grouped $P$ value of oral azithromycin.

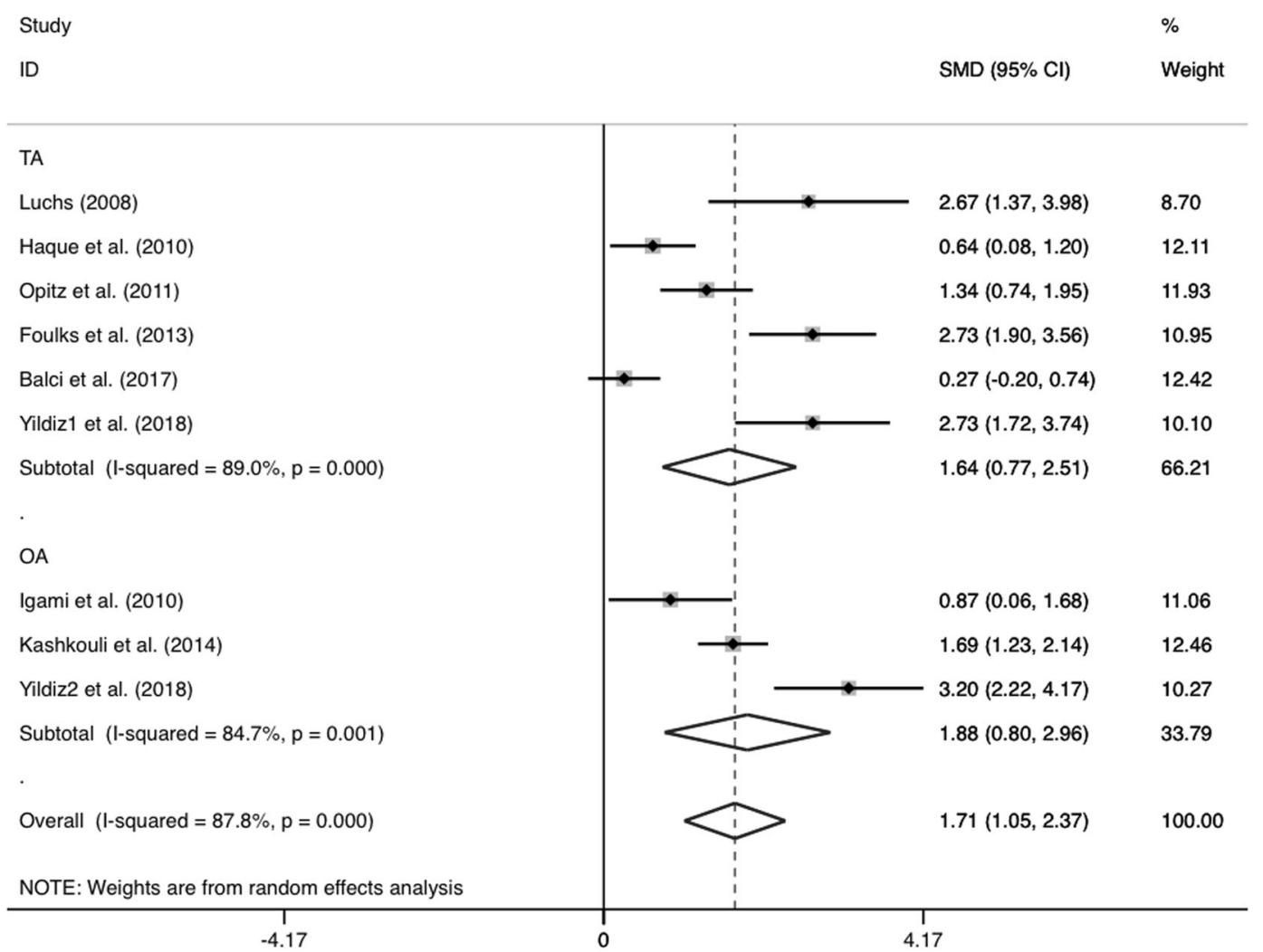

Fig. 5 Forest plot of subgroups showing the effect of Azithromycin on plugging of the meibomian gland in MGD patients (TA or OA), ordered by date of publication. Plugging of the meibomian gland significantly improved after administering either TA or OA. 


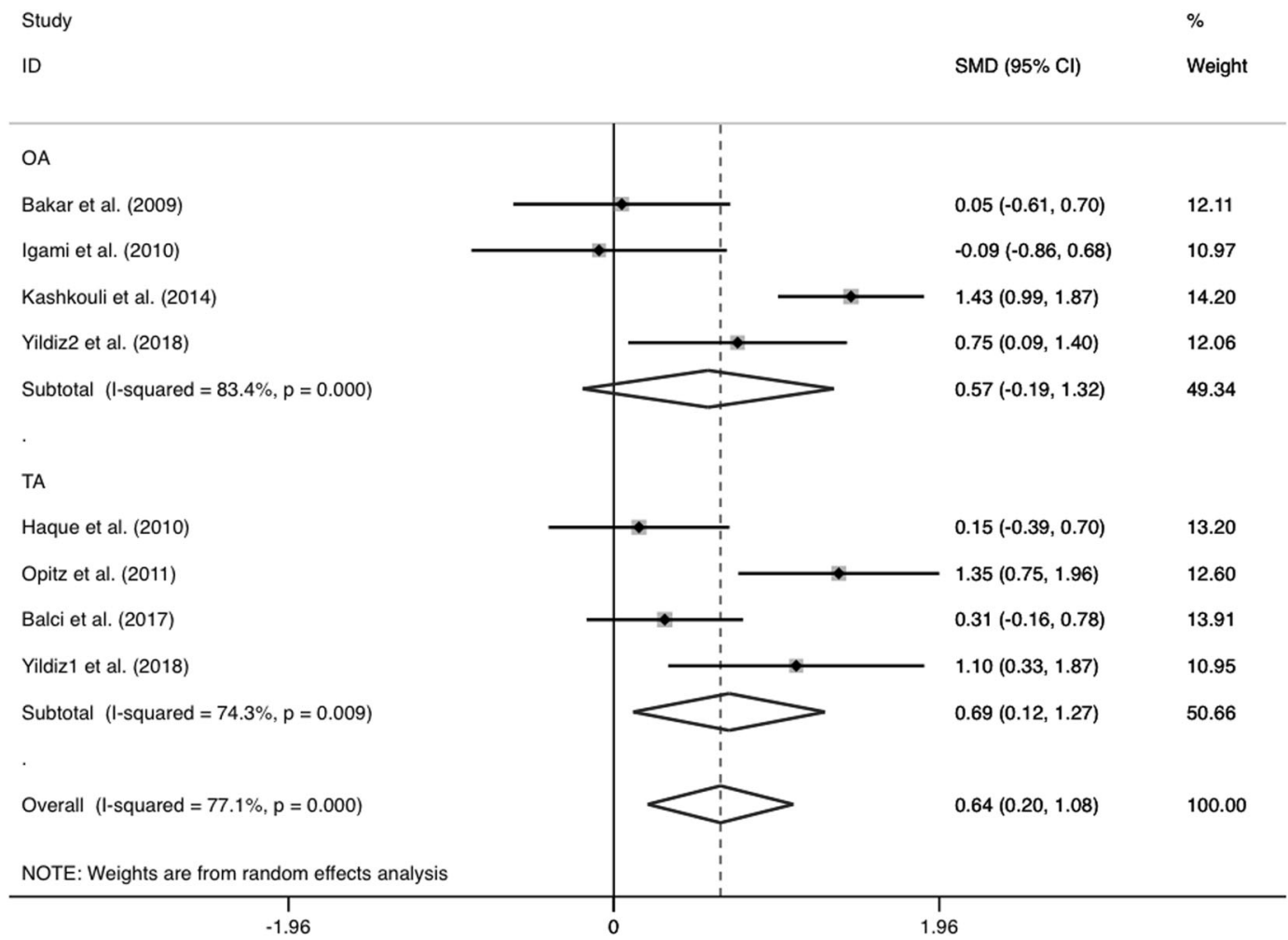

Fig. 6 Forest plot of subgroups showing the effect of Azithromycin on corneal staining in MGD patients (OA or TA), ordered by date of publication. The combined corneal staining after applying TA significantly improved compared with the baseline, but not after administering OA.

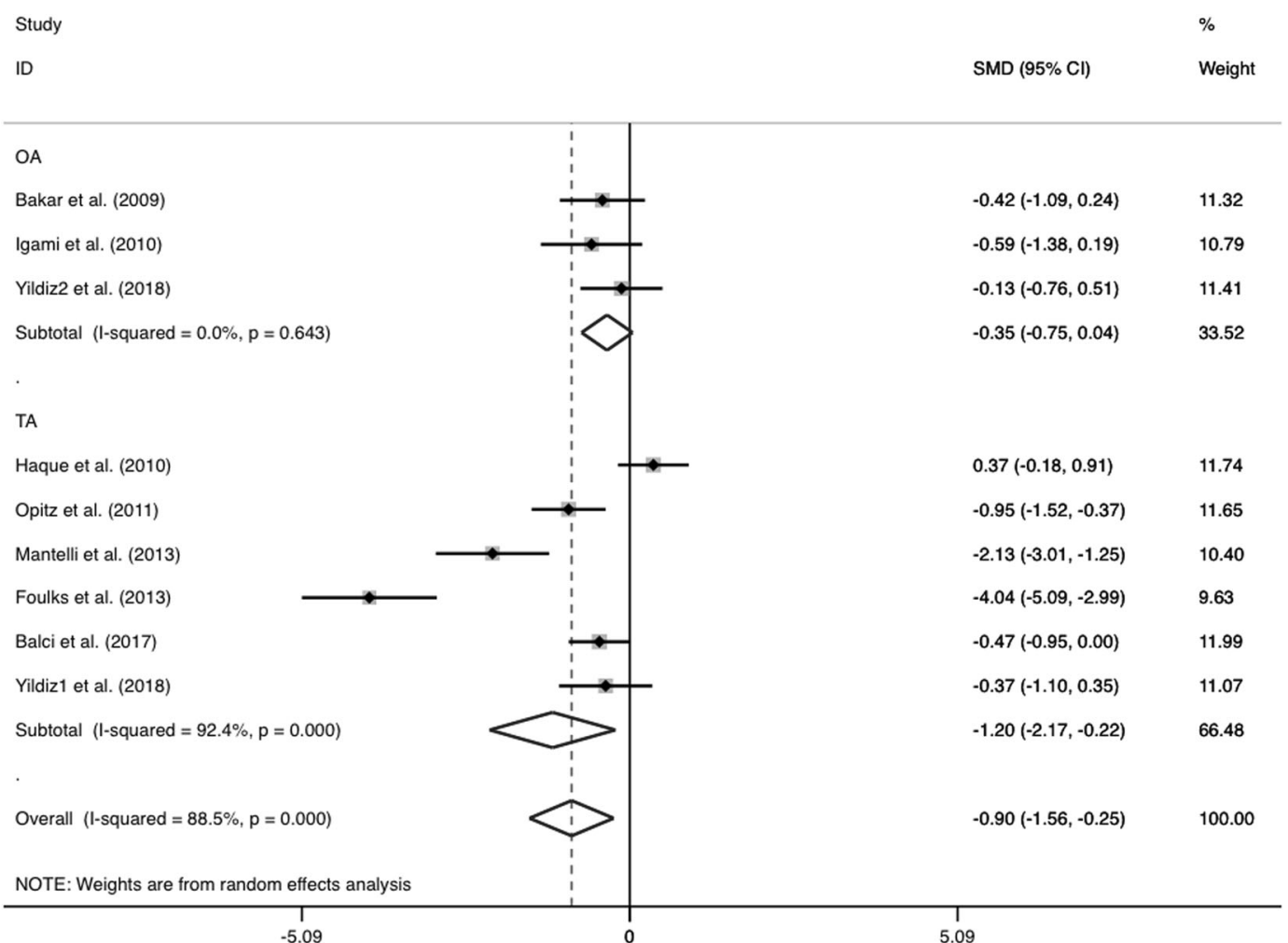

Fig. 7 Forest plot of subgroups showing the effect of Azithromycin on the quality of tear film in MGD patients (OA or TA), ordered by date of publication. The pooled TBUT increased distinctly from the baseline after applying TA but not after administering OA. 


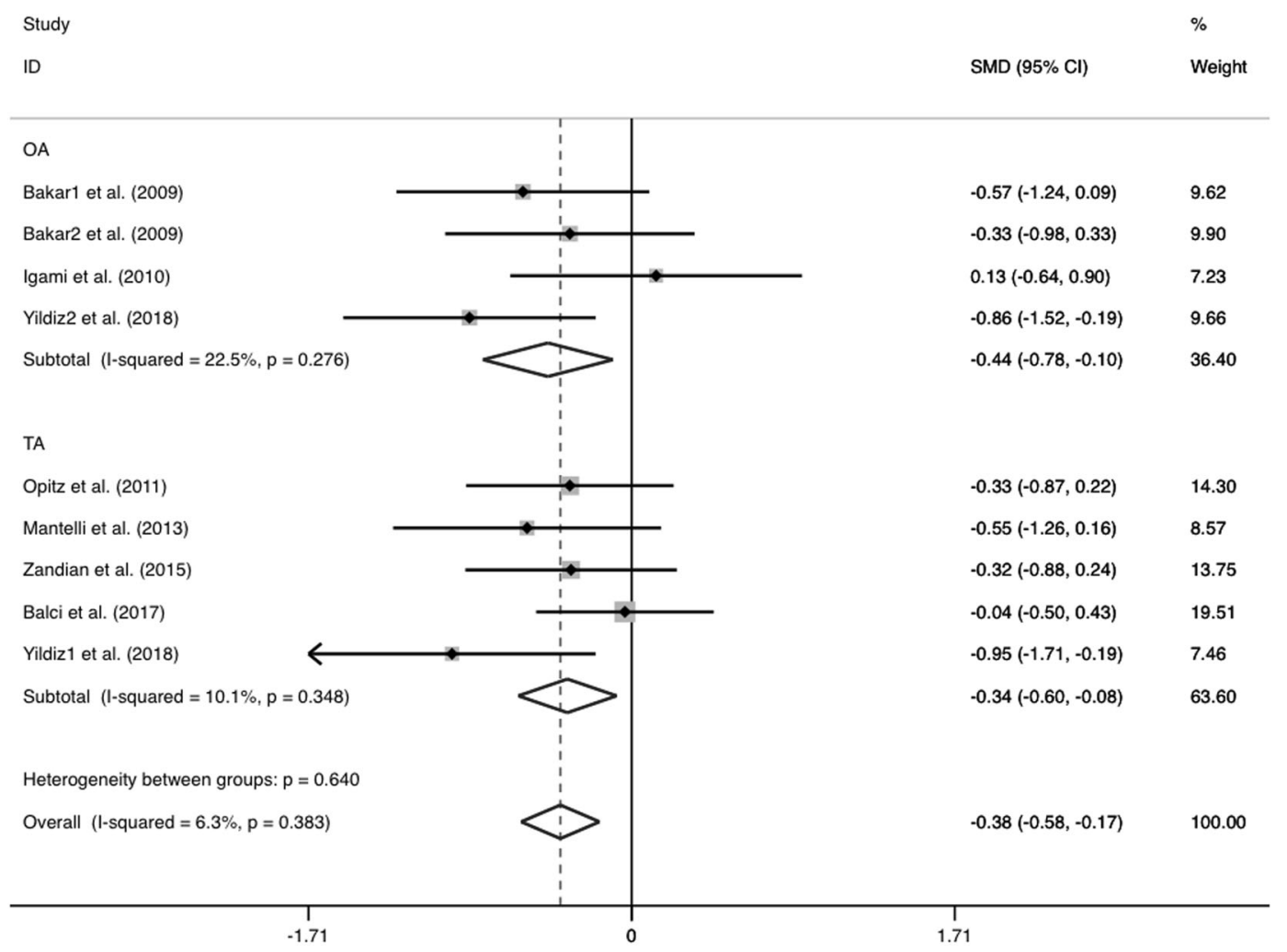

Fig. 8 Forest plot of subgroups showing the effect of Azithromycin on tear secretion in MGD patients (OA or TA), ordered by date of publication. Tear secretion distinctly increased after applying both TA and OA.

\section{Occurrence rate of adverse events after administering azithromycin}

The combined rate of adverse events after applying TA was 25\% (95\% CI: $0.19-0.31$ ). The common adverse events reported in these studies were all related to ocular discomfort, including irrigation sensation, ocular redness, and blurred vision [4, 17, 24]. No systemic complications were reported after TA treatment. In addition, the pooled rate was 7\% (95\% CI: $0.02-0.13$ ) after OA administration. Gastro-intestinal upset and decreased appetite were common complaints, but severe systemic complications were not reported in these studies [14, 19, 22].

\section{Discussion}

In this meta-analysis, the studies of treating MGD with azithromycin were systematically reviewed, and the clinical outcomes were pooled to demonstrate the effectiveness of azithromycin to manage MGD with respect to symptom scores, eyelid signs, and objective measurements. The pooled results showed that either OA or TA could significantly alleviate subjective symptoms and improve eyelid signs, plugging of the meibomian gland, meibum quality, and conjunctival injection. These results proved the effectiveness of treating MGD with azithromycin.

Previous studies have demonstrated that an antiinflammatory property was one aspect of azithromycin to treat MGD [27]. The antibiotic could eliminate inflammation at different levels. On the cellular level, the antibiotic could mediate neutrophil apoptosis and suppress their migration and phagocytic function [28]. An experimental study also showed it had the effects of inhibiting macrophage and dendritic cell migration in a mouse model of a cornea burn [29]. On the molecular level, azithromycin has effects on reducing pro-inflammatory cytokines/chemokines and metalloproteinases, such as IL-8, IL-1, TNF- $\alpha$, nitric oxide, prostaglandin E2, MMP-1, and MMP-9, via its antioxidative and antibacterial properties (reducing exotoxininduced cytokines) $[9-11,28,30]$. In addition, it may downregulate growth-related oncogene- $\alpha$ and suppress nuclear transcription factor NFkB and Toll-like receptors in vitro $[27,31]$.

Bacterial colonization also plays an important role in the development of MGD [32]. Some authors revealed S. aureus, Staphylococcus epidermidis, Propionibacterium acnes, and Corynebacterium were more frequently isolated from patients with posterior blepharitis than from healthy patients [33]. In addition, these bacteria can aggravate the inflammation of the posterior eyelid margin and change the meibum properties by 
releasing lipolytic enzymes, which could change the lipids to free fatty acids to irritate the ocular surface [34]. Azithromycin is a broad-spectrum antibiotic that can suppress bacteria effectively through binding to the $50 \mathrm{~S}$ subunit of the bacterial ribosome and inhibiting RNA synthesis [25]. Given that mechanism, the antibacterial effects exerted by azithromycin is the other aspect to managing MGD.

Although symptom scores and eyelid signs could be improved significantly after administering either OA or TA, the combined data in this study representing corneal staining and TBUT were improved distinctly by applying TA but not by administering OA. The results suggested that different formulations of azithromycin have varied abilities to improve the quality of tear film. Theoretically, the therapeutic effects after applying TA should be more direct, and the time to demonstrate the effects should also be less than the time for OA. This hypothesis was proven by Yildiz et al., who found that longer cytological improvements and better stabilization of tear film occurred after applying TA instead of administering OA [1]. However, another study demonstrated administering OD for 2 months could achieve comparable effects of applying TA for 1 month with respect to TBUT and corneal staining [20], suggesting prolonged therapy of OA/OD should be adopted to improve the quality of tear film distinctly. Moreover, our pooled results showed tear production was increased significantly by either OA or TA. Some authors assumed that the anti-inflammatory effects of azithromycin on accessory lacrimal glands could increase the aqueous secretion [16].

In this meta-analysis, we also combined the studies on treating MGD using both TA and OD to compare the effectiveness when considering the latter is also a potent agent to treat MGD and ocular rosacea. Interestingly, our pooled results showed that there were no significant differences between the two therapies regarding symptom score, tear secretion and TBUT, but the therapeutic courses of OD were obviously longer than those of TA in these studies. Foulks et al. investigated the changes in meibum composition through spectroscopic analysis after applying the two agents and found they had different mechanisms of managing MGD [20]. Although the pooled results showed OD was not inferior to TA to treat MGD, only prolonged therapy of OD could achieve comparable improvement in the quality of tear film [20]. Similarly, Zandian et al. proved OD was superior to TA in reducing eye redness and plugging of the meibomian gland in the same course (3 weeks) [21]. Given that result, TA appeared to reach therapeutic effects more rapidly, but the costs for TA are higher than the costs of OD in the US market [21]. Occurrence rates of adverse events after administering TA and OA were also pooled in this study. Although the rate seemed to be higher for TA $(25 \%)$ than for OA (7\%), no systemic adverse events were reported after using TA [4, 17, 24], which suggests TA may be safer and more suitable for children or child-bearing women. Systemic adverse events caused by OA were mainly related to gastro-intestinal upset, which was tolerable by most of the patients [14, 19, 22]. Considering that result, OA is cheaper and more convenient to obtain for most adult patients with MGD who have no special restraints.

To be honest, differences among these studies regarding therapeutic regimens (dose, frequency, and course), supplemental management, and follow-up periods may bias the pooled results, which was reflected by the distinct heterogeneities. One inevitable factor to invalidate the conclusion drawn from this meta-analysis is most of the participants also employed artificial tears, lid hygiene, and warm compresses as the routine treatment when they were administered azithromycin, which might confound the effects of the medication to treat MGD. To some extent, the supplemental treatment may also improve the stability of tear film, eyelid swelling, plugging of the meibomian gland, meibum quality, and corneal staining. Considering these positive effects yielded by the supplemental treatment, further studies should avoid these confounding factors in order to make a confirmative conclusion. Small sample sizes, different study designs and variance of severities of the studies might also influence the validity of this meta-analysis. In addition, we were unable to conduct further comparisons between OA and TA and investigate the relationship between effectiveness and doses further because of the nature of the data. However, based on the current evidence provided by this study, the effectiveness of treating MGD with either TA or OA was basically confirmed. Randomized, multiple centered and large sample size studies should be carried out to explore the best regimens to treat MGD, and the safety of azithromycin for the long term should also be observed.

In conclusion, meibomian gland dysfunction could be treated effectively with oral or topical azithromycin by improving symptoms, clinical signs, and stabilization of the tear film. Topical azithromycin seemed to be superior over oral azithromycin or doxycycline in improving the quality of tear film in the short term.

\section{Compliance with ethical standards}

Conflict of interest The authors declare that they have no conflict of interest.

Publisher's note Springer Nature remains neutral with regard to jurisdictional claims in published maps and institutional affiliations.

\section{References}

1. Yildiz E, Yenerel NM, Turan-Yardimci A, Erkan M, Gunes P. Comparison of the clinical efficacy of topical and systemic 
azithromycin treatment for posterior blepharitis. J Ocul Pharm Ther. 2018;34:365-72.

2. Nichols KK, Foulks GN, Bron AJ, Glasgow BJ, Dogru M, Tsubota K, et al. The international workshop on meibomian gland dysfunction: executive summary. Invest Ophthalmol Vis Sci. 2011;52:1922-9.

3. Huber-Spitzy V, Baumgartner I, Böhler-Sommeregger K, Grabner G. Blepharitis-a diagnostic and therapeutic challenge. A report on consecutive cases. Graefes Arch Clin Exp Ophthalmol. 1991;229:224-7.

4. Balci O, Gulkilik G. Assessment of efficacy of topical azithromycin 1.5 per cent ophthalmic solution for the treatment of meibomian gland dysfunction. Clin Exp Optom. 2018;101:18-22.

5. Henriquez AS, Korb DR. Meibomian glands and contact lens wear. Br J Ophthalmol. 1981;65:108-11.

6. Perry HD, Doshi-Carnevale S, Donnenfeld ED, Solomon R, Biser SA, Bloom AH. Efficacy of commercially available topical cyclosporine A $0.05 \%$ in the treatment of meibomian gland dysfunction. Cornea. 2006;25:171-5.

7. Rubin M, Rao SN. Efficacy of topical cyclosporin $0.05 \%$ in the treatment of posterior blepharitis. J Ocul Pharm Ther. 2006;22:47-53.

8. AlHity A, Lockington D. Oral azithromycin as the systemic treatment of choice in the treatment of meibomian gland disease. Clin Exp Ophthalmol. 2016;44:199-201.

9. Tabbara KF, al-Kharashi SA, al-Mansouri SM, al-Omar OM, Cooper H, el-Asrar AM, et al. Ocular levels of azithromycin. Arch Ophthalmol. 1998;116:1625-8.

10. Qiao J, Yan X. Emerging treatment options for meibomian gland dysfunction. Clin Ophthalmol. 2013;7:1797-803.

11. Borchman D, Yappert MC, Foulks GN. Changes in human meibum lipid with meibomian gland dysfunction using principal component analysis. Exp Eye Res. 2010;91:246-56.

12. Bakar O, Demircay Z, Toker E, Cakir S. Ocular signs, symptoms and tear function tests of papulopustular rosacea patients receiving azithromycin. J Eur Acad Dermatol Venereol. 2009;23:544-9.

13. Igami TZ, Holzchuh R, Osaki TH, Santo RM, Kara-Jose N, Hida RY. Oral azithromycin for treatment of posterior blepharitis. Cornea. 2011;30:1145-9.

14. Greene JB, Jeng BH, Fintelmann RE, Margolis TP. Oral azithromycin for the treatment of meibomitis. JAMA Ophthalmol. 2014;132:121-2.

15. Haque RM, Torkildsen GL, Brubaker K, Zink RC, Kowalski RP, Mah FS, et al. Multicenter open-label study evaluating the efficacy of azithromycin ophthalmic solution $1 \%$ on the signs and symptoms of subjects with blepharitis. Cornea. 2010;29:871-7.

16. Opitz DL, Tyler KF. Efficacy of azithromycin $1 \%$ ophthalmic solution for treatment of ocular surface disease from posterior blepharitis. Clin Exp Optom. 2011;94:200-6.

17. Doan S, Gabison E, Chiambaretta F, Touati M, Cochereau I. Efficacy of azithromycin $1.5 \%$ eye drops in childhood ocular rosacea with phlyctenular blepharokeratoconjunctivitis. J Ophthalmic Inflamm Infec. 2013;3:38.

18. Lazreg S. Role of Azithromycin in the treatment of children meibomian gland disease. Invest Ophthalmol Vis Sci. 2014;55:1501.
19. Mantelli F, Di Zazzo A, Sacchetti M, Dianzani C, Lambiase A, Bonini S. Topical azithromycin as a novel treatment for ocular rosacea. Ocul Immunol Inflamm. 2013;21:371-7.

20. Foulks GN, Borchman D, Yappert M, Kakar S. Topical azithromycin and oral doxycycline therapy of meibomian gland dysfunction: a comparative clinical and spectroscopic pilot study. Cornea. 2013;32:44-53.

21. Zandian M, Rahimian N, Soheilifar S. Comparison of therapeutic effects of topical azithromycin solution and systemic doxycycline on posterior blepharitis. Int J Ophthalmol. 2016;9:1016-9.

22. Kashkouli MB, Fazel AJ, Kiavash V, Nojomi M, Ghiasian L. Oral azithromycin versus doxycycline in meibomian gland dysfunction: a randomised double-masked open-label clinical trial. $\mathrm{Br} \mathrm{J}$ Ophthalmol. 2015;99:199-204.

23. Heitz A, Arnaud S, Merklen C, Cribier B, Speeg-Schatz C, Bourcier T. Oral azithromycin versus doxycyclin for the treatment of moderate to severe meibomian gland dysfunction (MGD) - a randomized prospective non-inferiority study. Invest Ophthalmol Vis Sci. 2014;55:1481.

24. Luchs J. Efficacy of topical azithromycin ophthalmic solution $1 \%$ in the treatment of posterior blepharitis. Adv Ther. 2008;25:858-70.

25. Fadlallah A, Rami HE, Fahd D, Dunia I, Bejjani R, Chlela E, et al. Azithromycin $1.5 \%$ ophthalmic solution: efficacy and treatment modalities in chronic blepharitis. Arq Bras Oftalmol. 2012;75:178-82.

26. Doan S, Baudouin C, Labetoulle M, Bourcier T, Hoffart L, Pisella $\mathrm{PJ}$, et al. Efficacy and safety of azithromycin $1.5 \%$ eye drops $\left(\right.$ Azyter $^{\circledR}$ ) in patients with moderate to severe chronic blepharitis. Invest Ophthalmol Vis Sci. 2014;55:47.

27. Aghai ZH, Kode A, Saslow JG, Nakhla T, Farhath S, Stahl GE, et al. Azithromycin suppresses activation of nuclear factor-kappa $\mathrm{B}$ and synthesis of pro-inflammatory cytokines in tracheal aspirate cells from premature infants. Pediatr Res. 2007;62:483-8.

28. Amar T, Caillaud T, Elena PP. Ocular pharmacokinetic study following single and multiple azithromycin administrations in pigmented rabbits. Curr Eye Res. 2008;33:149-58.

29. Sadrai Z, Hajrasouliha AR, Chauhan S, Saban DR, Dastjerdi MH, Dana R. Effects of topical azithromycin on corneal innate immune responses. Invest Ophthalmol Vis Sci. 2011;52:2525-31.

30. Liu Y, Kam WR, Ding J, Sullivan DA. Can tetracycline antibiotics duplicate the ability of azithromycin to stimulate human meibomian gland epithelial cell differentiation? Cornea. 2015;34:342-6.

31. Akpek EK, Vittitow J, Verhoeven RS, Brubaker K, Amar T, Powell KD, et al. Ocular surface distribution and pharmacokinetics of a novel ophthalmic $1 \%$ azithromycin formulation. J Ocul Pharm Ther. 2009;25:433-9.

32. Iovieno A, Lambiase A, Micera A, Stampachiacchiere B, Sgrulletta R, Bonini S. In vivo characterization of doxycycline effects on tear metalloproteinases in patients with chronic blepharitis. Eur J Ophthalmol. 2009;19:708-16.

33. Dougherty JM, Mcculley JP. Comparative bacteriology of chronic blepharitis. Br J Ophthalmol. 1984;68:524-8.

34. Mcculley JP, Dougherty JM. Bacterial aspects of chronic blepharitis. Trans Ophthalmol Soc UK. 1986;105:314-8. 